\title{
Transition between two ferromagnetic states driven by orbital ordering in $\mathrm{La}_{0.88} \mathrm{Sr}_{0.12} \mathrm{MnO}_{3}$
}

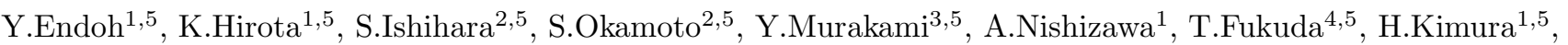 \\ H.Nojiri $^{2,5}$, K.Kaneko ${ }^{2,5}$, and S.Maekawa ${ }^{2,5}$ \\ ${ }^{1}$ Department of Physics, Tohoku University, Sendai, 980-8578 Japan \\ ${ }^{2}$ Institute for Materials Research, Tohoku University, Sendai, 980-8577 Japan \\ ${ }^{3}$ Photon Factory, Institute of Materials Structure Science, KEK, Tsukuba 305-0801, Japan \\ ${ }^{4}$ Department of Synchrotron Radiation Facility Project, JAERI, Sayo 679-5143, Japan \\ ${ }^{5}$ CREST, Japan Science and Technology Corp., Tsukuba 305-0047, Japan
}

(August 18, 2019)

\begin{abstract}
A lightly doped perovskite mangantite $\mathrm{La}_{0.88} \mathrm{Sr}_{0.12} \mathrm{MnO}_{3}$ exhibits a phase transition at $T_{O O}=$ $145 \mathrm{~K}$ from a ferromagnetic metal $\left(T_{C}=172 \mathrm{~K}\right)$ to a novel ferromagnetic insulator. We identify that the key parameter in the transition is the orbital degree of freedom in $e_{g}$ electrons. By utilizing the resonant x-ray scattering technique, orbital ordering is directly detected below $T_{O O}$, in spite of a significant diminution of the cooperative Jahn-Teller distortion. The new experimental features are well described by a theory treating the orbital degree of freedom under strong electron correlation. The present experimental and theoretical studies uncover a crucial role of the orbital degree in the metal-insulator transition in lightly doped manganites.
\end{abstract}

PACS numbers: 75.30.Vn, 71.30.+h, 75.30.Et, 71.10.-w

Colossal magnetoresistance (CMR), recently discovered in perovskite manganites, occurs in the vicinity of metal-insulator (MI) transition. It was proposed many years ago that the double-exchange (DE) mechanism plays an essential role to realize the ferromagnetic metallic state in doped manganites [1,2]. However, the CMR effects cannot be explained within this simple concept [3] and additional ingredients, such as lattice distortion, electron correlation, and orbital degree of freedom, are stressed. To reveal the mechanism of the MI transition and its mutual relation to CMR, it is essential to study the lightly doped regime in detail, where several phase boundaries are entangled.

In $\mathrm{La}_{1-x} \mathrm{Sr}_{x} \mathrm{MnO}_{3}$ around $x \sim 0.12$, the temperature dependence of electrical resistivity shows metallic behavior below $T_{C}$ consistent with the DE picture. As temperature decreases further, however, it shows a sharp upturn below a certain temperature [4], defined $T_{O O}$ in the present paper. Note that a transition from ferromagnetic metallic (FM) state to the ferromagnetic insulating (FI) state occurs at $T_{O O}$. Kawano et al. 5 | revealed by neutron diffraction that $\mathrm{La}_{0.875} \mathrm{Sr}_{0.125} \mathrm{MnO}_{3}\left(T_{C}=230 \mathrm{~K}\right)$ exhibits successive structural phase transitions; hightemperature pseudo-cubic phase $\left(\mathrm{O}^{*}: a \sim b \sim c / \sqrt{2}\right)$ to intermediate Jahn-Teller (JT) distorted orthorhombic phase $\left(\mathrm{O}^{\prime}: b>a \gg c / \sqrt{2}\right)$ at $T_{H}=260 \mathrm{~K}$ and to low-temperature $\mathrm{O}^{*}$ phase at $T_{O O}=160 \mathrm{~K}$. Here, we use orthorhombic Pbnm notation. These complicated behaviors are far beyond the simple DE scenario.

In this Letter, we report that the MI transition in $\mathrm{La}_{0.88} \mathrm{Sr}_{0.12} \mathrm{MnO}_{3}$ is actually driven by orbital ordering, which was directly observed by the recently developed decisive technique, i.e., the resonant x-ray scattering [6,7]. It is counter-intuitive that the orbital ordering appears in the FI phase where a long-range cooperative JT distortion significantly diminishes [8]. As discussed later, this orbital ordering can be realized by the super-exchange (SE) process under strong electron correlation together with ferromagnetic ordering, not necessarily associated with a cooperative JT distortion. This ferromagnetic SE interaction coexists with the DE interaction which facilitates carrier mobility above $T_{O O}$. The transition from FM to FI can be induced by applying a magnetic field [9 11]. Our theoretical calculation, where the orbital degree and the electron correlation are considered, well reproduces these unconventional experimental results in $\mathrm{La}_{0.88} \mathrm{Sr}_{0.12} \mathrm{MnO}_{3}$.

We have grown series of single crystals by the floatingzone method using a lamp image furnace. Typical mosaicness measured by neutron diffraction is less than $0.3^{\circ}$ FWHM, indicating that the samples are highly crystalline. We have carried out neutron-diffraction of an $x=0.12$ single crystal using the TOPAN triple-axis spectrometer in the JRR-3M reactor in JAERI. As shown in Fig. 1, we found successive structural phase transition and magnetic ordering consistent with Ref. [5], though transition temperatures are different reflecting a slight discrepancy in the hole concentration, i.e., $x=0.12$ and 0.125 . However, high-resolution synchrotron $\mathrm{x}$ - ray powder diffraction by Cox et al. [12] on a carefully crushed small crystal from the same batch reveals that the intermediate phase is monoclinic and that the lowtemperature phase is triclinic though the distortion is extremely small. An electron-diffraction study by Tsuda et al. 13 using the same batch gave consistent results. Note that typical $\mathrm{x}$-ray peak widths are $\sim 0.01^{\circ}$, close to the instrumental resolution. This indicates that the $d$ spacing distribution is negligible, which is another proof of high quality of our samples.

Here, we briefly mention the charge ordering proposed by Yamada et al [14]. We have indeed confirmed the superlattices below $\bar{T}_{O O}$ by neutron and x-ray scatterings. 


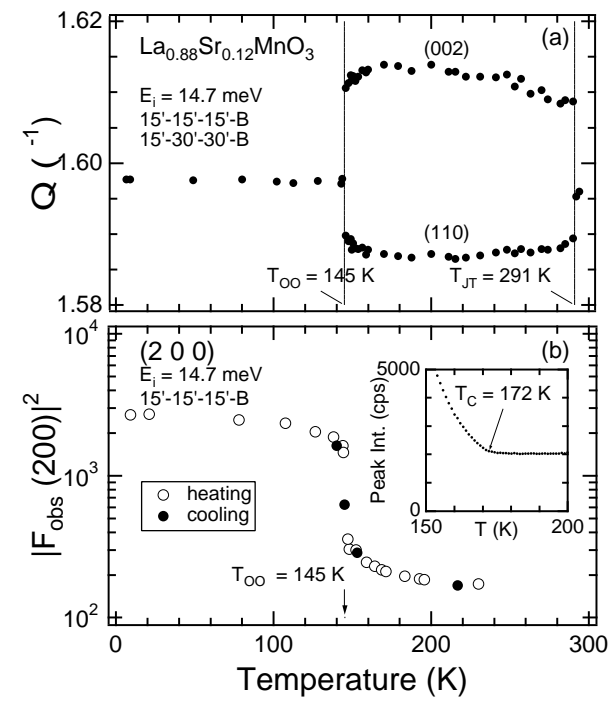

FIG. 1. Temperature dependence of (a) lattice parameter, and integrated intensities of (b) (2 00 ) ferromagnetic Bragg reflection measured with $14.7 \mathrm{meV}$ neutrons.

In the x-ray study, however, the energy dependence of the superlattice peak around the Mn K-edge does not show a resonance feature 15, 16] which is a characteristic in $\mathrm{Mn}^{3+} / \mathrm{Mn}^{4+}$ charge ordering $[6]$. We thus conclude that, below $T_{O O}$, there appears a long-range structural modulation along the $c$ - axis though neither a conventional charge ordering nor a long-range cooperative JT distortion as seen in the $\mathrm{O}^{\prime}$ phase exist.

Ferromagnetic ordering below $T_{C}=172 \mathrm{~K}$ was observed by neutron diffraction as shown in Fig. Il(b). With further decreasing temperature, the $\left(\begin{array}{lll}2 & 0 & 0\end{array}\right)$ peak exhibits a discontinuous increase at temperature corresponding to $T_{O O}$, where the structural phase transition shown in Fig. 1(a) occurs. We found that magnetic Bragg peaks appear at $\left(\begin{array}{lll}0 & 0 & l\end{array}\right)(l=$ odd $)$ below $T_{O O}$ indicating antiferromagnetic component. When the magnetic structure below $T_{O O}$ is interpreted as a canted structure, the canting angle is, however, small and the FI state is a good approximation.

The orbital states were observed by synchrotron x-ray diffraction measurements on four-circle spectrometers at beamlines $4 \mathrm{C}$ and $16 \mathrm{~A} 2$ in the Photon Factory in KEK. We have tuned the incident energy near the Mn K- edge $(6.552 \mathrm{KeV})$. The $\left(\begin{array}{lll}0 & 1 & 0\end{array}\right)$ plane of a $\mathrm{La}_{0.88} \mathrm{Sr}_{0.12} \mathrm{MnO}_{3}$ single crystal $(\sim 2 \phi \times 2 \mathrm{~mm})$ from the same batch, which was carefully polished, was aligned within the scattering plane.

Figure 2(a) shows the incident energy dependence of (0 30 ) peak, which is structurally forbidden, at $12 \mathrm{~K}$. The peak exhibits a sharp enhancement at the Mn K-edge, determined experimentally from fluorescence. As discussed in $\mathrm{La}_{1.5} \mathrm{Sr}_{0.5} \mathrm{MnO}_{4}$ and also in $\mathrm{LaMnO}_{3}$ [6], the appearance of such a forbidden peak is considered as a signature of orbital ordering of $\mathrm{Mn}^{3+} e_{g}$ electrons: Orbital ordering gives rise to anisotropy in the anomalous scattering

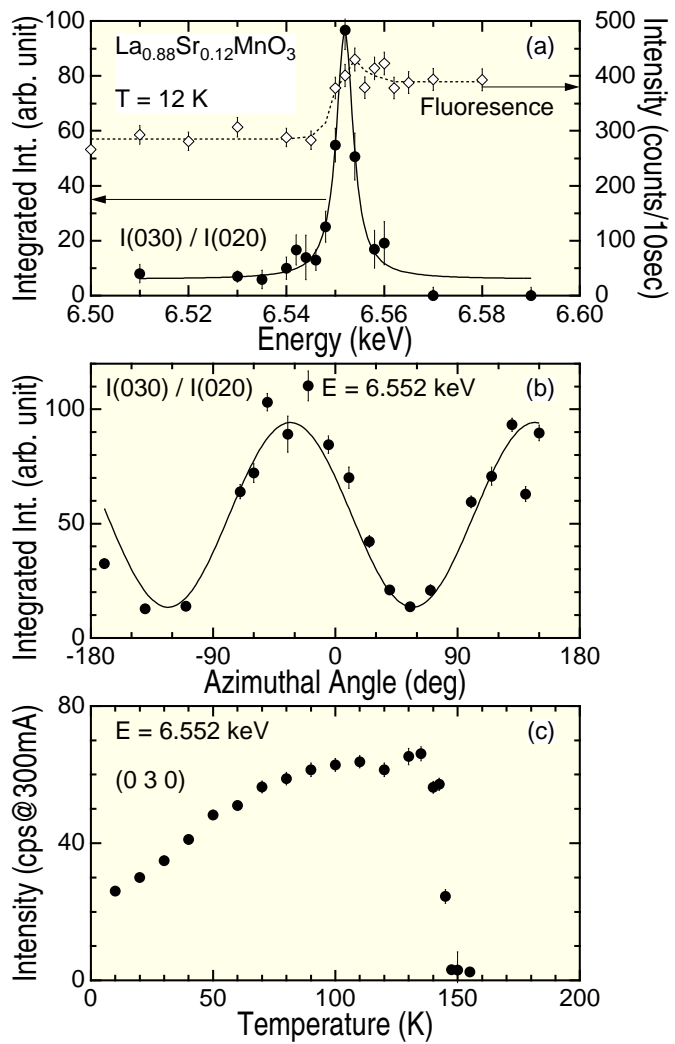

FIG. 2. (a) Energy dependence of intensity of the orbital-ordering reflection $\left(\begin{array}{lll}0 & 3 & 0\end{array}\right)$ at $T=12 \mathrm{~K}$. The dashed curve represents fluorescence showing the resonant energy (6.552 KeV) corresponding to the Mn K edge. (b) The azimuthal angle dependence of ( $\left.\begin{array}{lll}0 & 3 & 0\end{array}\right)$. The solid line is two-fold squared sine curve of angular dependence. (c) Temperature dependence of (lllll $\left.\begin{array}{lll}0 & 3\end{array}\right)$ peak intensity.

factor, which is enhanced and thus visible near the Mn Kedge. The antiferro(AF)-type orbital ordering is directly confirmed by rotating the crystal around the scattering vector kept at $\left(\begin{array}{lll}0 & 3 & 0\end{array}\right)$, i.e., azimuthal scan. Fig. 2(b) shows the azimuthal scan, clearly revealing square of sinusoidal angle dependence of two-fold symmetry.

Although the orbital ordering in $\mathrm{La}_{0.88} \mathrm{Sr}_{0.12} \mathrm{MnO}_{3}$ seems similar to that of $\mathrm{LaMnO}_{3}$, there is a marked difference. As shown in Fig. 2(c), the orbital ordering appears only below $T_{O O}=145 \mathrm{~K}$, where the cooperative JT distortion disappears or significantly decreases. On the other hand, in $\mathrm{LaMnO}_{3}$ where the orbital ordering appears in the JT distorted orthorhombic phase [6], it has been believed that long- range arrangement of JT distorted $\mathrm{MnO}_{6}$ octahedra facilitates $d_{3 x^{2}-r^{2}} / d_{3 y^{2}-r^{2}}$ type orbital ordering. The orbital ordering is consistent with the spin-wave dispersion reported by Hirota et al. [17], who proposed the dimensional crossover in lightly doped $\mathrm{La}_{1-x} \mathrm{Sr}_{x} \mathrm{MnO}_{3}$ [18]. The spin dynamics of $\mathrm{La}_{1-x} \mathrm{Sr}_{x} \mathrm{MnO}_{3}$ drastically changes from two-dimenional state as seen in $\mathrm{LaMnO}_{3}$, due to the AF-type orbital ordering of $d_{3 x^{2}-r^{2}} / d_{3 y^{2}-r^{2}}$, to three-dimensional isotropic 
ferromagnetic state around $x \approx 0.1$. Therefore, we anticipate that $\mathrm{La}_{0.88} \mathrm{Sr}_{0.12} \mathrm{MnO}_{3}$ should have a different orbital state, e.g., the hybridization of $d_{z^{2}-x^{2}\left(y^{2}-z^{2}\right)}$ and $d_{3 x^{2}-r^{2}\left(3 y^{2}-r^{2}\right)}$. Note that the intensity of $\left(\begin{array}{lll}0 & 3 & 0\end{array}\right)$ resonant peak is significantly reduced at low temperatures compared with that just below $T_{O O}$, indicating that the AF- type orbital ordering becomes reduced with decreasing temperature. This reduction is not necessarily due to the instability of orbital ordering at low temperatures because the gradual change of type of the orbital ordering , e.g., AF-type to ferro-type, gives rise to the effect.

Now we theoretically reveal the microscopic mechanism of the newly found experimental features. The spin and orbital states are investigated by utilizing the model Hamiltonian where the spin and orbital degrees of freedom are treated on an equal footing together with the strong electron correlation [19]: $\mathcal{H}=\mathcal{H}_{t}+\mathcal{H}_{J}+\mathcal{H}_{H}+$ $\mathcal{H}_{A F}$. The first and second terms correspond to the socalled $t$ - and $J$-terms in the $t J$-model for $e_{g}$ electrons. These are given by [20] $\mathcal{H}_{t}=\sum_{\langle i j\rangle \gamma \gamma^{\prime} \sigma} t_{i j}^{\gamma \gamma^{\prime}} \tilde{d}_{i \gamma \sigma}^{\dagger} \tilde{d}_{j \gamma^{\prime} \sigma}+$ H.c. and

$$
\begin{aligned}
\mathcal{H}_{J}= & -2 J_{1} \sum_{\langle i j\rangle}\left(\frac{3}{4} n_{i} n_{j}+\vec{S}_{i} \cdot \vec{S}_{j}\right)\left(\frac{1}{4}-\tau_{i}^{l} \tau_{j}^{l}\right) \\
& -2 J_{2} \sum_{\langle i j\rangle}\left(\frac{1}{4} n_{i} n_{j}-\vec{S}_{i} \cdot \vec{S}_{j}\right)\left(\frac{3}{4}+\tau_{i}^{l} \tau_{j}^{l}+\tau_{i}^{l}+\tau_{j}^{l}\right),
\end{aligned}
$$

where $\tau_{i}^{l}=\cos \left(\frac{2 \pi}{3} n_{l}\right) T_{i z}-\sin \left(\frac{2 \pi}{3} n_{l}\right) T_{i x}$ and $\left(n_{x}, n_{y}, n_{z}\right)=$ $(1,2,3)$. $l$ denotes a direction of a bond connecting $i$ and $j$ sites. $\tilde{d}_{i \gamma \sigma}$ is the annihilation operator of $e_{g}$ electron at site $i$ with spin $\sigma$ and orbital $\gamma$ with excluding double occupancy. The spin and orbital states are denoted by the spin operator $\vec{S}_{i}$ and the pseudo-spin operator $\vec{T}_{i}$, respectively. The latter describes which of the orbitals is occupied. The third and fourth terms in the Hamiltonian describe the Hund coupling: $\mathcal{H}_{H}=-J_{H} \sum_{i} \vec{S}_{t i} \cdot \vec{S}_{i}$ and the AF magnetic interaction between $t_{2 g}$ spins: $\mathcal{H}_{A F}=$ $J_{A F} \sum_{\langle i j\rangle} \vec{S}_{t i} \cdot \vec{S}_{t j}$, respectively, where $\vec{S}_{t i}$ is the spin operator for $t_{2 g}$ electrons with $S=3 / 2$. Since the cooperative JT distortion has been experimentally found to be weak around $x \sim 0.1$, the electron-lattice coupling is neglected in the model. As seen in the first term of $\mathcal{H}_{J}$, the ferromagnetic SE interaction results from the orbital degeneracy and the Hund coupling between $e_{g}$ electrons 21 23]: Through the coupling between spin and orbital degrees in $\mathcal{H}_{J}$, the ferromagnetic ordering and AF-type orbital ordering are cooperatively stabilized.

The mean field approximation is adopted in the calculation of the spin and orbital states at finite $x$ and $T$ 24. Two kinds of the mean field for $\vec{S}_{i}$ and $\vec{T}_{i}$ are introduced. The both states are described by the distribution functions and the mean fields are optimized by minimizing the free energy. The ferromagnetic spin and G-type pseudo-spin alignments are assumed. The detailed formulation will be presented elsewhere.

The calculated phase diagram is presented in Fig. 3.

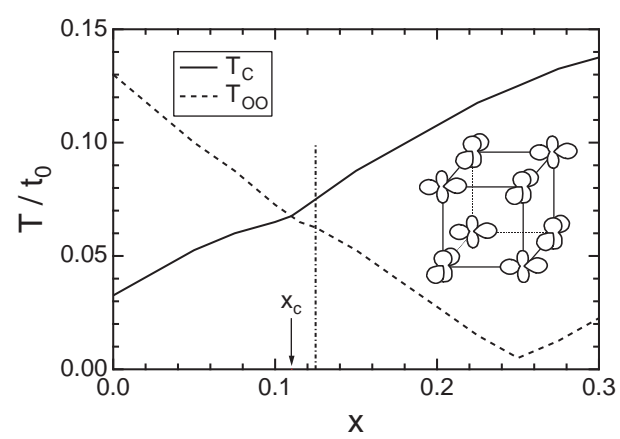

FIG. 3. The spin and orbital phase diagram as a function of carrier concentration $(x)$ and temperature $(T)$. The straight and broken lines are for the spin and orbital ordering temperatures. The experimental data are compared with the theoretical results at $x$ shown by the vertical dotted line. The inset shows the schematic picture of the orbital structure below $T_{O O}$ at $x=0.125$. $\left[\left(\theta_{A} / \theta_{B}=-\theta_{A}\right)\right.$ where $\left.\theta_{A}=\pi / 2\right]$. The parameter values are chosen to be $J_{1} / t_{0}=0.25, J_{2} / t_{0}=0.0625$, and $J_{A F} / t_{0}=0.004$.

With doping of holes, a leading magnetic interaction gradually turns from the SE interaction in the lower $x$ to the DE one 25]. $T_{C}$ monotonically increases with increasing $x$. On the other hand, the orbital state changes (1)from the AF-type ordering favored by $\mathcal{H}_{J}$ to the ferrotype ordering induced by $\mathcal{H}_{t}$ due to the gain of the kinetic energy. Thus, $T_{O O}$ decreases with doping of holes. In the undoped insulator, $T_{O O}$ is higher than $T_{C}$ because the interaction between orbitals $\left(3 J_{1} / 2\right)$ are larger than that between spins $\left(-J_{1} / 2\right)$, as expected from the first term in $\mathcal{H}_{J}$. Consequently, $T_{C}$ and $T_{O O}$ cross with each other at $x_{c} \sim 0.1$ as seen in Fig. 3 .

We next focus on the region where $x$ is slightly higher than $x_{c}$. There are two kinds of the ferromagnetic phase; the phase between $T_{C}$ and $T_{O O}$ and that below $T_{O O}$. In the high-temperature phase, the orbital is disordered. In the low-temperature phase, on the other hand, the AF-type orbital ordering appears and the SE interaction is enhanced through the spin-orbital coupling in $H_{J}$. Since the AF-type ordering reduces the kinetic energy, the DE interaction is weakened. Consequently, the metallic character is degraded. We identify the low- and high-temperature phases to be FI and FM in $\mathrm{La}_{0.88} \mathrm{Sr}_{0.12} \mathrm{MnO}_{3}$, respectively. Note again that the AF-type orbital ordering is driven by the electronic mechanism, not supported by the JT distortion 26. The orbital ordering (the inset of Fig. 3) is denoted as $\left(\theta_{A} / \theta_{B}=-\theta_{A}\right)$ with $\theta_{A}=\pi / 2$, where $\theta_{A(B)}$ is the angle in the orbital space in the $A(B)$ sublattice. This is the mixture of $d_{z^{2}-x^{2}\left(y^{2}-z^{2}\right)}$ and $d_{3 x^{2}-r^{2}\left(3 y^{2}-r^{2}\right)}$, which is consistent with the isotropic ferromagnetic spin wave dispersion. The characteristic curve in the azimuthal angle dependence of the resonant x-ray scattering (Fig. 2(b)) is reproduced by this type of the ordering [7]. The coupling between spin and orbital reflects on the temperature dependence of the magnetization. It is shown in Fig. 1(a), 

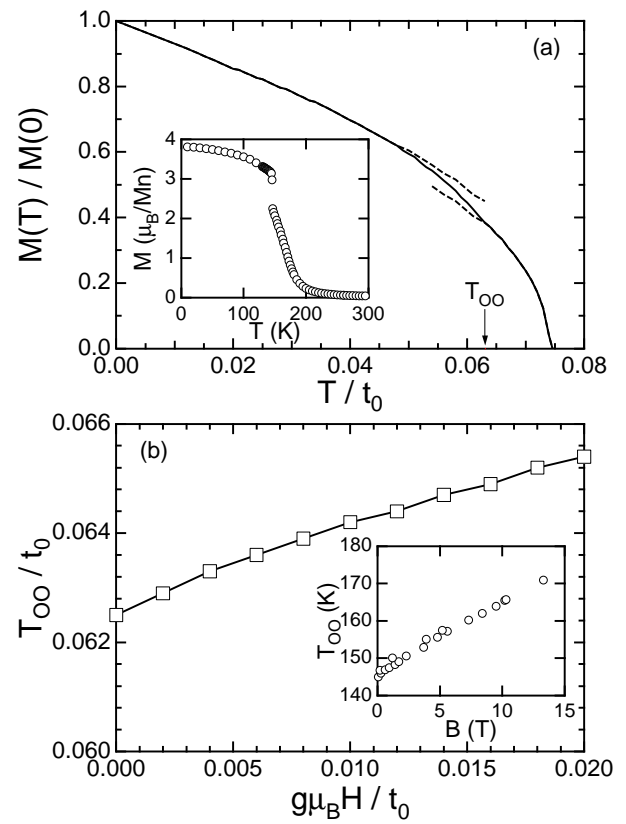

FIG. 4. (a) Temperature dependence of the calculated magnetization at $x=0.125$. The inset shows the magnetization curve in $\mathrm{La}_{0.88} \mathrm{Sr}_{0.12} \mathrm{MnO}_{3}$ at $B=0.5 T$. (b) Field dependence of the orbital ordering temperature. The inset is obtained from the electrical resistivity and the magnetization in $\mathrm{La}_{0.88} \mathrm{Sr}_{0.12} \mathrm{MnO}_{3}$ 11]. Parameter values are the same with those in Fig. 3 .

that the magnetization is enhanced below $T_{O O}$. The calculated result is consistent with the experimental observation in $\mathrm{La}_{0.88} \mathrm{Sr}_{0.12} \mathrm{MnO}_{3}$ (the inset of Fig. A(a)). This is a strong evidence of the novel coupling between spin and orbital degrees.

In Fig. 4 (b), TOO is shown as a function of the applied magnetic field. The applied magnetic field stabilizes the low-temperature ferromagnetic phase accompanied with the orbital ordering. This is because the ferromagnetic spin correlation induced by the field enhances the interaction between orbitals through the spin-orbital coupling in $\mathcal{H}_{J}$. In other word, the magnetic field controls the orbital states. The theoretical $T_{O O}$ versus $H$ curve qualitatively reproduces the experimental results in $\mathrm{La}_{0.88} \mathrm{Sr}_{0.12} \mathrm{MnO}_{3}$ (the inset of Fig. 4(b)) and strongly supports that the orbital degree plays a key role in the low temperature phase and the transition at $T_{O O}$.

To conclude, the transition from the ferromagnetic metallic to the ferromagnetic insulating phases in $\mathrm{La}_{0.88} \mathrm{Sr}_{0.12} \mathrm{MnO}_{3}$ is ascribed to the transition of orbital order-disorder states. The orbital ordering is observed in the low temperature phase where the cooperative Jahn-Teller type distortion is significantly diminished. The stability of the two phases are controlled by changing temperature and/or applying magnetic field, and the unique coupling between spin and orbital degrees is found. The present investigation shows a novel role of the orbital degree of freedom as a hidden parameter in the MI transition in lightly doped CMR manganites.

Authors acknowledge D. E. Cox, K. Tsuda, and T. Inami for their valuable discussions. Part of the numerical calculation was performed in the HITACS-3800/380 superconputing facilities in IMR, Tohoku University. S.O. acknowledges the financial support of JSPS Research Fellowships for Young Scientists.

[1] C. Zener, Phys. Rev. 82, 403 (1951).

[2] P. W. Anderson, and H. Hasegawa, Phys. Rev. 100, 675 (1955).

[3] A. J. Millis et al., Phys. Rev. Lett. 77, 175 (1996).

[4] A. Urushibara et al., Phys. Rev. B 51, 14103 (1995).

[5] H. Kawano et al., Phys. Rev. B 53, R14709 (1996).

[6] Y. Murakami et al., Phys. Rev. Lett. 80, 1932 (1998), and Phys. Rev. Lett. 81, 582 (1998).

[7] S. Ishihara and S. Maekawa, Phys. Rev. Lett. 80, 3799 (1998), and Phys. Rev. B 5813449 (1998).

[8] D. N. Argyriou et al., Phys. Rev. Lett. 763826 (1996).

[9] R. Senis et al., Phys. Rev. B 57,14680 (1998).

[10] S. Uhlenbruck et al., Phys. Rev. Lett. 82, 185 (1999).

[11] H. Nojiri et al., (unpublished).

[12] D. E. Cox et al., (unpublished).

[13] K. Tsuda et al., (unpublished).

[14] Y. Yamada et al., Phys. Rev. Lett. 77, 904 (1996).

[15] T. Inami et al., (to be published in Jpn. J. Appl. Phys.)

[16] T. Fukuda et al., (unpublished).

[17] K. Hirota et al., Physica B 237-238, 36 (1997).

[18] K. Hirota et al., J. Phys. Soc. Jpn. 65, 3736 (1996). See also F. Moussa et al., Phys. Rev. B 54, 15149 (1996).

[19] S. Ishihara et al., Phys. Rev. B 55, 8280 (1997).

[20] $t_{i j}^{\gamma \gamma^{\prime}}$ is the transfer intensity between $i$ and $j$ sites with $\gamma$ and $\gamma^{\prime}$ orbitals. $J_{1}=t_{0}^{2} /\left(U^{\prime}-I\right)$ and $J_{2}=t_{0}^{2} /\left(U^{\prime}+\right.$ $\left.I+2 J_{H}\right)$, where $t_{0}$ is the orbital independent transfer intensity, $U^{\prime}$ is the Coulomb interaction between different orbitals and $I$ is the exchange interaction between $e_{g}$ electrons. $J_{1}>J_{2}$ is satisfied.

[21] J. B. Goodenough, Phys. Rev. 100, 564 (1955).

[22] J. Kanamori, J. Phys. Chem. Solids 10, 87 (1959).

[23] K. I. Kugel, and D. I. Khomskii, ZhETF Pis. Red. 15 , 629 (1972), (JETP Lett. 15, 446 (1972)).

[24] P. G. de Gennes, Phys. Rev. 118, 141 (1960).

[25] R. Maezono et al., Phys. Rev. B 57, R13993 (1998).

[26] Two kinds of the ferromagnetic phase driven by the DE interaction and the JT distortion are discussed in S. Yunoki et al., Phys. Rev. Lett. 81, 5612 (1998). 